\title{
Progress towards therapies for disease modification in Parkinson's disease
}

Nirosen Vijiaratnam, FRACP ${ }^{1,2}$, Tanya Simuni, $\mathrm{MD}^{3}$, Oliver Bandmann, $\mathrm{PhD}^{4}$, Huw R Morris, $\mathrm{PhD}^{1,2}$, Thomas Foltynie, $\mathrm{PhD}^{1,2}$

1. Department of Clinical and Movement Neurosciences, UCL Queen Square Institute of Neurology, London, UK.

2. The National Hospital for Neurology and Neurosurgery, Queen Square, London, UK.

3. Parkinson's Disease and Movement Disorders Center, Northwestern University Feinberg School of Medicine, Chicago, IL, USA.

4. Sheffield Institute for Translational Neuroscience (SITraN), University of Sheffield, Sheffield, UK.

Corresponding author:

Prof Thomas Foltynie

Department of Clinical and Movement Neurosciences,

UCL Queen Square Institute of Neurology,

London, UK.

Email: t.foltynie@ucl.ac.uk

\begin{abstract}
The development of interventions to slow or halt the progression of Parkinson's disease (PD) remains the major priority for people affected with the illness and PD researchers alike. To date, there have been no agents shown to have unequivocal evidence of disease modifying effects in PD. This may relate not only to inadequate approaches to the selection of
\end{abstract}


therapeutic candidates, but also to insufficient attention to detail in clinical trial design. Better understanding of PD pathogenesis, associated with advances in laboratory models, the use of objective biomarkers of disease progression and target engagement, and a focus on agents known to be safe for human use, alongside the use of precision medicine approaches should together, greatly increase the likelihood for successful identification of disease modifying treatments for PD.

\section{Search strategy and selection criteria}

A literature search was conducted in January 2021, using the following electronic databases: Medline (Ovid), PubMed and Scopus. The terms 'Parkinson's disease*' AND 'disease modifying treatments' or 'therapy*' were used. Articles describing clinical trials of disease modifying treatments in patients with Parkinson's disease were identified and abstracts were reviewed for relevance. No language restrictions were imposed. Although no specific time limit was imposed for when trials were published, clinical trials conducted in the last 5 years and of specific value in highlighting the core objectives of this review were prioritised.

\section{Introduction}

Parkinson's disease (PD) has an increasing prevalence, with projections of an increase to around 10 million people globally by $2030 .{ }^{1}$ People with PD experience a combination of motor (bradykinesia, rigidity, and tremor) and non-motor features (including constipation, hyposmia, depression, cognitive decline, and sleep alterations $)^{2}$ which substantially affect their quality of life and caregiver needs. Despite the availability of symptomatic treatment options for PD, ultimately patients will develop progressive disability and loss of independence as a 
result of disease progression. These medical, social and economic concerns highlight the urgent need for effective treatments that modify the course of PD and maintain or improve patient quality of life.

None of the approaches tried to date have yet translated to a clinically proven disease modifying treatment. In this review we explore the reasons that may explain this, highlighting that until recently we have selected therapeutic candidates based on unrepresentative preclinical models, and performed trials without properly considering heterogenous PD subgroups and mechanisms of confirmation of target engagement at optimal dosage. Specifically, we focus on 1) trials with recently reported negative results, to examine possible explanations for their failure, 2) examples of approaches that have used more novel and innovative trial approaches to help highlight the concept of disease modification and address the challenges of demonstrating this in $\mathrm{PD}, 3$ ) trials that are still ongoing and awaiting definitive results. We have not attempted to provide an exhaustive description of all ongoing clinical trials (for an exhaustive review of disease modifying trials in PD see $^{3}$ ), but instead have highlighted examples of trials that are potentially instructive for readers with an interest in the development of disease modifying approaches in PD. Increasing recognition of the importance of these issues allows growing confidence that better therapeutic candidates are being selected and tested in appropriate subgroups of patients with PD, which can thus be predicted to have far greater chances of successfully modifying the course of PD.

\section{Reasons for past failures of disease modifying trials in PD}

A large number of trials have attempted to evaluate the potential for disease modification using drugs with a diverse range of mechanisms of action. Unfortunately, the majority of these have either failed to demonstrate clinical benefit or have provided inconclusive results, despite 
convincing evidence of potential efficacy in preclinical studies. Reasons for failure vary according to each agent but include poor selection of agents due to insufficiently representative preclinical models of PD, failure of an agent to reach and engage its target in adequate doses, inadequacy in trial design and the possible confounding by symptomatic effects, insufficient duration of follow up, and lack of objective measures of true clinical disease progression.

Failure of a drug to influence disease progression in a broad population of patients may simply be the result of failure to recruit the correct patient subtype. For example, agents considered to be primarily acting on mitochondrial function (eg Co-enzyme Q10) may only be of use in patients with impaired mitochondrial function. Similarly, GDNF administration may only be helpful among those patients expressing the GDNF (ret) receptor which may be facilitated by combination treatment with Nurr $1 .^{4}$ Recent improvements in our understanding of PD pathophysiology, its relationship with individual clinical variability, methods used in preclinical testing, and how we might measure PD progression and therapeutic target engagement has increased the likelihood of developing therapies with strong prospects for disease modification.

\section{Considering PD heterogeneity}

Substantial advances in our understanding of PD pathogenesis have emerged in the identification of molecular pathways implicated in the pathogenesis of familial PD also being aberrant in sporadic cases. From this emerges a key concept regarding whether PD "subtypes" are in fact unique biological entities or reflect a disease spectrum with substantial mechanistic overlap. ${ }^{5}$ This concept is fundamentally important for disease modifying approaches, in that subtypes of PD may be considered according to different triggers for the disease, which may be individually amenable to different "upstream" interventions, while common mechanisms 
related to PD progression may provide scope for agents with broad "downstream" rescue effects irrespective of the original trigger.

The mechanistic heterogeneity of PD is increasingly recognized - for example, lysosomal dysfunction may be a prominent and early event in some but not all patients. ${ }^{6} \mathrm{~A}$ further aspect to patient stratification that may gain increasing relevance is the variability of individual gut microbiome characteristics though the extent to which this may be cause or effect in PD pathogenesis requires further validation. ${ }^{7}$ Any neuroprotective intervention may therefore be more likely to succeed if a "precision medicine" approach is applied. This emphasizes the need for improved tools to undertake biological stratification prior to recruitment. For some agents this may substantially reduce the number of eligible participants, while for other downstream interventions, e.g. intervening on alpha synuclein toxicity or propagation, the majority of individuals with clinically diagnosed PD may still remain eligible. See Figure 1.

\section{Targeted preclinical testing}

Before initiating human clinical trials, any drug must demonstrate robust effects at the preclinical stage. Traditional animal models of PD have focused on recapitulating the loss of dopaminergic neurons and therefore exploited toxins to invoke neuronal damage analogous to that seen in PD in rodents or non-human primates through the local administration of the neurotoxin 6-hydroxydopamine (6-OHDA) or systemic administration of the neurotoxin 1-methyl-4-phenyl-1,2,3,6-tetrahydropyridine (MPTP). Although adequate at replicating PD motor features, these models do not exhibit the extra dopaminergic and non-motor dysfunction seen in early PD nor do they exhibit the presence of Lewy bodies or the progressive neurodegenerative process of human PD. ${ }^{8}$

Transgenic animals with monogenic mutations seen in familial PD have provided a vastly more representative model of human PD pathogenesis. These include transgenic mice 
overexpressing normal or mutated human $\alpha$-synuclein, ${ }^{9}$ mice in which Tfam - the gene encoding mitochondrial transcription factor $\mathrm{A}$ is knocked out resulting in disrupted mitochondrial function ${ }^{10}$ and mouse models where a single injection of synthetic misfolded $\alpha$-synuclein initiates a cascade of neurodegenerative events, leading to Lewy body-type inclusions, selective nigral neuronal loss and motor impairments. ${ }^{11,12}$ The increasing use of induced pluripotent stem cells or induced neurons derived from skin biopsies taken from patients with PD, allows further assessment of drug effects in preventing neurodegeneration. ${ }^{13,14,15}$ Even more representative models might exploit the use of organoids that can model an entire basal ganglia network. ${ }^{16}$ Although these models do not yet perfectly reproduce the complex in vivo pattern of human PD, they have enabled progress towards the discovery of potential targets of disease-modifying strategies with greater confidence. The choice of which model to use in preclinical drug assessment depends on the putative mechanism of action of the intervention e.g. an alpha synuclein based intervention should show efficacy in a relevant alpha synuclein based model. By extension, evidence of beneficial effects of an agent in laboratory/animal models of mitochondrial dysfunction should prompt exploration of clinical effects (in the first instance) in PD patients with evidence of ongoing mitochondrial dysfunction. When assessed for its potential as a disease modifying agent in PD, rasagiline had shown evidence of beneficial effects in 6-OHDA and MPTP rodent models, suggesting a potential mechanism of action via oxidative stress and mitochondrial dysfunction ${ }^{17}$, but at the clinical trial design stage, the potential relevance of subtypes of PD, as well as the importance of confirmation of target engagement may not have been as well recognised. ${ }^{18}$

\section{Target engagement and clinical outcomes}


Ahead of large, expensive clinical efficacy trials, there is a need to accurately measure drug engagement in salient pathways, at which point the optimal dose, and its safety/tolerability can be determined. This also serves as an opportunity to confirm whether predictions regarding which subtype of patients are responders are accurate. Many of the agents that have thus far 'failed' in clinical trials may have fared better if re-assessed using a more sophisticated approach to patient selection and enrichment, and outcome measurement.

While clinical and quality of life measures are essential as the primary outcomes for phase 3 trials, the emphasis at the earlier stages of assessment should shift towards more sensitive markers of disease progression such as structural imaging, ${ }^{19}$ or biological measures of target engagement such as CSF alpha synuclein, ${ }^{20}$ or ideally by non-invasive means of measuring alpha synuclein pathology e.g. by using functional imaging. ${ }^{21}$ This approach can be further tailored according to the choice of the disease modifying approach. For example, an agent considered to engage with lysosomal function should be accompanied by measurement of enzyme activity in blood/ CSF, ${ }^{22}$ while mitochondrial approaches (eg rasagiline) might exploit MR spectroscopy to confirm target engagement. ${ }^{23}$

Taken together, our understanding of the pathophysiological processes of PD compel us to use far better ways of documenting the potential of disease modifying approaches in people with PD, both during the preclinical phase and in phase 2 evaluations including a clear and objective means of confirming target engagement with a candidate drug. During phase 3 evaluation, outcome measurement must be relevant to patients e.g. to evaluate impact on function, activities of daily living and quality of life. These aspects will require long term follow up especially given the likely small effect sizes anticipated. To improve the signal to noise ratio among these outcomes, and thus power to detect small effect sizes, the development of objective and accurate quantification of motor and non-motor symptom severity through 
wearable devices and other remote technologies is of major importance. ${ }^{24,25}$ Panel 1 discusses further concepts relevant to the conduct of disease modifying trials in PD.

\section{Panel 1 - General concepts relevant for disease modification trials}

\section{Symptomatic verses Disease Modifying}

It can be argued that any drug that effectively improves the control of symptoms of PD (whether related to motor control, autonomic or cognitive dysfunction, or mood) could be disease modifying as it will likely allow an increase in healthy behaviours such as exercise, or reduce the risk of injury due to falls. For the purposes of this review however, we choose to consider as disease modifying, only those agents which

- have a demonstrable effect on the initial triggers that lead to neuronal degeneration,

- allow better neuronal compensatory responses or

- reduce the propagation or progress of pathological changes of PD through the brain/ body.

It is entirely possible that an effective disease modifying agent may have both symptomatic and disease modifying effects and allow better functioning of individual neurons, brain networks and the whole patient, in tandem with engagement with a process of cell survival/ escape from the toxic processes associated with PD. Trials to evaluate disease modification must be designed to take account for the potential confounds of improved symptom control.

Multiple attempts have previously been made to assess disease modification properties of treatments that are now largely considered purely symptomatic. In the ELLDOPA trial, ${ }^{26} 3$ doses of levodopa were compared to placebo over a short follow-up period. As the UPDRS motor scores did not decline in the levodopa-treated groups to the level of the placebo group 
even after a 4-week washout, this finding was argued to be due to a neuroprotective effect. This is now thought to be due to the long duration symptomatic effect of levodopa as confirmed by the recent findings of the LEAP-study, largely dispelling the notion of disease modification by levodopa. ${ }^{27}$ This emphasises the importance of trial design in the objective quantification of disease progression. Delayed start designs, washout designs, randomized withdrawal designs have all been considered, ${ }^{28}$ as have long term designs with multi-modal clinical outcomes. ${ }^{29}$ See Figure 2 for a description of these different methodological approaches.

An alternative approach would be to shift the focus away from terminology such as 'symptomatic' or 'disease modifying' and focus purely on describing the slowing of clinical outcomes regardless of the explanation. ${ }^{30}$ An example of this could be indicating that a given intervention results in reduced worsening of PD features (measured by e.g. UPDRS) over 24 months or that fewer individuals developed disease milestones such as falls or cognitive impairment over the predefined period. Avoiding the need for definitive evidence of neuroprotection, could facilitate more rapid approval of agents that have demonstrated efficacy in studies.

\section{Earlier verses longer?}

It is also likely that disease-modifying effects will be modest in magnitude in comparison to the symptomatic effects of dopaminergic replacement, which therefore requires further consideration of the population of patients to select for trials.

- Earlier disease (i.e. pre levodopa replacement) will be more amenable to disease modifying interventions, however disease progression limits the duration of follow up possible in newly diagnosed populations of patients before dopaminergic replacement is required. 
- The major challenges of PD relate to the dopa refractory elements, i.e. the swallowing, balance and cognitive issues which occur in the more advanced stages of the disease, therefore there may be a sufficient window to intervene to prevent the development of these issues even in the period after dopaminergic medication initiation, which may facilitate longer term follow up in later phase trials.

- Recruiting people who have already started levodopa replacement and assessing them in the "Practically defined Off medication" state is possible but makes assumptions that the long duration effects of levodopa are consistent within and between patients.

\section{Current therapies being evaluated for disease modification}

Potential therapeutic targets for PD disease modification can be broadly considered under categories directly targeting alpha-synuclein itself, "upstream" factors that can trigger alphasynuclein pathology or "downstream" mechanisms related to the spread of pathology or that may facilitate neuronal compensatory responses, though substantial interplay between these categories occur. The approaches discussed here are not exhaustive but prioritise areas in which there has been the most research activity recently and on our own consideration of what we believe to be the most promising approaches moving forward. Tables 1 and 2 provide a summary of approaches currently being explored in PD and their stages in the development process.

\section{Agents targeting specific molecular pathways}

\section{Alpha-synuclein}


While aggregated alpha-synuclein is not strictly necessary nor sufficient for the development of neurodegeneration or clinical parkinsonism, ${ }^{31}$ it is clearly of major potential relevance as evidenced by rare patients with PD carrying duplications of the alpha synuclein gene. ${ }^{32}$ Nevertheless, the majority of PD patients' brains when examined at post mortem demonstrate several types of misfolded proteins, not just alpha synuclein, and unequivocal evidence that alpha synuclein aggregation is toxic rather than compensatory has not yet been established. This said, there appears to be a bidirectional relationship between specific toxic species of alpha synuclein and disturbances of the autophagy and lysosomal degradation pathways, dysfunction of the endoplasmic reticulum, mitochondria and Golgi apparatus, as well as disruptions of nuclear processes and synaptic-vesicle trafficking. ${ }^{2,33}$ Disturbance in these cellular pathways can lead to alpha-synuclein toxicity (disturbances of protein degradation and clearance) and therefore a self-perpetuating cycle of alpha-synuclein accumulation and further organelle dysfunction.

Another key aspect to consider is the propagation of alpha-synuclein pathology via cell to-cell transmission. This may occur in part through its secretion via exosome release and uptake via endocytosis. $^{34}$ Transmission of toxic conformations of alpha-synuclein are thought to subsequently induce seeding, recruitment, and permissive templating of normal alphasynuclein. This 'prion-like' spread of Lewy pathology is supported by several layers of evidence ${ }^{33,35}$ and proposed to begin outside the brain before reaching it via enteric and olfactory projections. ${ }^{36}$

Of major commercial interest therefore, are anti-alpha-synuclein antibodies, which comprise monoclonal antibodies targeting different parts of the alpha-synuclein protein and potentially enhance its immune mediated clearance while blocking its adverse cellular effects on neuronal processes by reducing cellular toxicity and pathological spread. ${ }^{37,38}$ Current approaches involve 
both active (PD01A, PD03A) ${ }^{39}$ and passive (BIIB054, BAN0805, PRX002/ RO7046015, MEDI1341, AF82422) ${ }^{40}$ immunization. Evidence for target engagement (serum free alphasynuclein level reduction and increased antibody-bound levels) and safety are promising ${ }^{41}$ and phase 2 randomized controlled clinical trials (BIIB054-SPARK trial; RO7046015PASADENA trial) are ongoing. These trials have included detailed imaging and a subset of patients have had CSF collection to help gauge target engagement.

Drug repurposing may also play a role. One example is the beta 2 -adrenoreceptor which is a potential regulator of the alpha-synuclein gene. The use of beta 2-adrenoreceptor agonists has been associated with a lower incidence of $\mathrm{PD}^{42}$ though the data have been inconsistent. ${ }^{43}$ There is evidence that beta agonists reduce alpha-synuclein gene transcription ${ }^{42}$ and early studies also seemed to suggest a symptomatic benefit ${ }^{44} 45$, although there are no trial data yet available to indicate disease modifying effects.

Therapeutic manipulation to induce autophagy of alpha-synuclein has been explored in PD, based on promising findings in disease models. ${ }^{46}$ Nilotinib is a non-receptor tyrosine kinase Abelson (c-Abl) inhibitor used for the treatment of chronic myeloid leukemia. c-Abl is a kinase present in most cells and involved in a variety of physiological functions including autophagy ${ }^{47}$ and becomes activated by cellular stress. Preclinical PD studies suggested nilotinib is effective in inducing autophagy and clearing alpha-synuclein ${ }^{48}$ while an unblinded study in individuals with PD dementia and dementia with Lewy bodies suggested potential clinical benefits, although with some concerns about the potential for cardiac arrhythmias. ${ }^{49}$ Two larger, placebo-controlled Phase II clinical trials "PD Nilotinib" and "Nilo-PD" aimed to explore these findings further though recently reported results suggest minimal CNS penetration of nilotinib effectively excluding the future potential for development of this drug. ${ }^{50,51}$ Additional CNS 
penetrant c-Abl inhibitors are currently in development (K-0706, ikt-148009). Preliminary studies on K-0706 have shown promise with excellent safety and tolerability and CSF penetration (https://www.clinicaltrials.gov/ct2/show/NCT02970019), and a Phase II study of 504 PD patients has recently commenced.

\section{LRRK2}

The most common cause of autosomal dominant PD (LRRK2-G2019S) results in increased LRRK2 kinase activity and disrupts autophagy. ${ }^{52}$ Furthermore, common variation at the LRRK2 locus is a risk factor for the development of PD. ${ }^{53}$ The toxic gain of function associated with LRRK2 mutations may lead to enhanced propagation of alpha synuclein mediated by several potential processes including the autophagy-lysosome system, mitochondrial function, phosphorylation of RAB proteins or interaction with protein $14-3-3 .{ }^{54}$ LRRK2 inhibitors have been developed and preclinical PD studies indicate they prevent neurodegeneration ${ }^{55}$, potentially by promoting autophagy. ${ }^{56}$ While early studies using LRRK2 inhibitors were limited by lung toxicity, two more LRRK2 inhibitors (DNL201 and DNL151) are currently being tested in phase $1 \mathrm{~b}$ studies. Clearly this approach is directed to addressing the underlying defect in a subset of people with PD due to LRRK2 mutations. It remains unclear whether the same mechanisms are recapitulated in patients with sporadic PD, and whether these potential therapies may have wider applicability. ${ }^{57}$

An alternative approach using an antisense oligonucleotide for LRRK2 inhibition is approaching clinical testing (BIIB094) on the back of promising data suggesting LRRK2 antisense oligonucleotides ameliorate alpha-synuclein aggregation in relevant preclinical 
models. ${ }^{58,59}$ Trial programs will be testing LRRK2 inhibition in LRRK2-associated and idiopathic PD. ${ }^{57}$

\section{Glucocerebrosidase}

Genetic variations in the GBA gene, which encodes the lysosomal enzyme $\beta$ glucocerebrosidase (GCase), are associated with Gaucher disease and PD. ${ }^{60}$ GBA-associated PD has a similar phenotype to idiopathic PD, though an earlier age at onset and more rapid cognitive decline has been noted in carriers. Normally, GCase is synthesized, folded and translocated to the lysosome, however mutant GCase remains in the endoplasmic reticulum with subsequent proteasome degradation and therefore reduced lysosomal levels. Subsequent decreased GCase activity alters glycosphingolipid homeostasis with further lysosomal dysfunction, aberrant vesicular transport, and alpha-synuclein aggregation. ${ }^{61}$

Ambroxol improves the translocation of mutant GCase thereby increasing GCase activity in cells carrying GBA mutations. ${ }^{62}$ The AiM-PD phase 2 trial recently reported promising findings in 18 participants with either GBA mutations or sporadic PD suggesting larger trials will be warranted. ${ }^{63}$ Most importantly, this trial included CSF analysis of GCase activity and demonstrated target engagement in those patients treated with Ambroxol.

Another small molecule LTI-291 is an activator of GCase with promising results (https://www.trialregister.nl/trial/6516; https://www.trialregister.nl/trial/7061) that may be heading for Phase II testing shortly. Further novel options currently in the preclinical development stages include GCase activators ${ }^{64}$ and gene therapy treatment for correcting GCase activity in GBA-associated PD (viral vector PR001). ${ }^{65}$ Measurements of GCase activity 
and glycosphingolipids in blood and serum will allow objective evaluation of target engagement and optimal dosing.

Substrate reduction therapy is also being explored to achieve normalisation of glycosphingolipid homeostasis. In the absence of normal GCase activity, glycolipids accumulate. Venglustat, Ibiglustat (or GZ/SAR402671) and GZ667161(brain-penetrant version), are oral inhibitors of the enzyme glucosylceramide synthase (GCS), which converts ceramide into glucosylceramide, a substrate of GCase that accumulates in GBA-associated disease. GCS inhibitors seem to reduce alpha-synuclein aggregation in PD models with and without GBA mutations. ${ }^{66}$ Different GBA mutations lead to different properties of the GCase protein therefore the success of some of these approaches may depend on further restricting recruitment to specific mutation carriers only ${ }^{67}$.

\section{Agents targeting specific neuronal rescue pathways}

\section{Calcium}

Specific neuronal populations with spontaneous pacemaking properties such as the SNc rely on L-type Cav1.3 Calcium channels, which promote calcium entry into the cell. ${ }^{68}$ Enhanced calcium entry is associated with increased oxidative stress, mitochondrial damage and cell death. Blockade of Cav1.3/Cav 1.2 L-type channels by the CNS penetrant dihydropyridine channel blocker, isradipine was shown to be neuroprotective in the dopamine toxin based animal models ${ }^{69}$ and had some support from epidemiological data. ${ }^{70}$ Nevertheless, no difference in MDS-UPDRS total scores after 36 months isradipine treatment was noted in a

recent phase 3 trial. $^{71}$ This discrepancy between positive preclinical data, supportive epidemiological data and negative human data may reflect insufficiently representative animal 
models, lack of human read out on target engagement, lack of precision of PD subtype, or simply inadequate dose selection (that was based on tolerability).

\section{Iron}

Iron overload in the SNc has been a consistent feature of PD post-mortem studies for decades. ${ }^{72}$ Recent detection of increased nigral iron content on sensitive MRI sequences has further affirmed this. ${ }^{73}$ Iron overload could potentially contribute to neuronal loss by increasing mitochondrial oxidative stress, followed by accumulation and aggregation of a-synuclein and neuronal apoptosis. Beneficial effects on PD progression using iron chelation with deferiprone have been reported when measured with the UPDRS as well as iron content measurement with T2* MR imaging. ${ }^{74}$ The objective measurement of target engagement will allow for comparison between clinical data and imaging changes. Results of a recently completed placebo-controlled study of 270 patients with deferiprone (FAIRPARK-II) are expected.

\section{Neuroinflammation}

The role of the immune system in exacerbating the neurodegenerative process is supported given that individuals with certain HLA alleles are linked with a risk of PD. ${ }^{75}$ Aggregated alpha-synuclein seems to modulate inflammatory responses via microglia, CD41 T cells and macrophages. ${ }^{76}$ Subsequent excessive activation of cytokines contributes to progressive neurodegeneration. ${ }^{77}$ Inflammation appears to be a relatively early feature of the disease,${ }^{76}$ and therefore is potentially a disease-modifying target over the entire disease course.

Sargramostim, a human recombinant granulocyte-macrophage colony- stimulating factor (GM- CSF) was assessed in a small group of PD patients and seemed to improve regulatory $\mathrm{T}$ cell function while arresting UPDRS motor score progression. ${ }^{78}$ The enzyme myeloperoxidase 
(MPO) has been of specific interest considering its well-established role in inflammation and degeneration mediated by microglial cells in PD. ${ }^{79}$ AZD3241, a selective and irreversible MPO inhibitor which suppresses microglia and protects dopaminergic neurons was well tolerated in phase I/II trials ${ }^{80,81}$ and found to effectively modulate the oxidative cellular environment using objective PET imaging. ${ }^{81}$

The activation of the microglial "NLR family pyrin domain containing 3" (NLRP3) inflammasome is implicated in PD with the accumulation of alpha-synuclein activating NLRP3. ${ }^{82}$ Inhibition or deficiency seems to rescue animal models ${ }^{83}$ and oral administration of a potent NLRP3 inhibitor has shown promise in inhibiting inflammasome activation, reducing alpha-synuclein accumulation, rescuing motor deficits and preventing neurodegeneration. ${ }^{84} \mathrm{~A}$ phase 1 trial exploring Inzomelid, a promising NLRP3 inhibitor recently completed recruitment. Standard safety and tolerability outcomes were measured though the secondary outcome of NLRP3 inhibition will be of particular interest.

Statins lower cholesterol levels by inhibiting 3-hydroxy-3-methylglutaryl-coenzyme A (HMGCoA) and simvastatin seems to have dopaminergic and motor behaviour benefits in rodents via a number of pathways though immune suppression appears to be a key aspect. ${ }^{85}$ Simvastatin $80 \mathrm{mg}$ daily as a potential PD disease modifying therapy is currently under evaluation in a phase II study. A potential concern is that only clinical outcome measures are being collected.

The activation of the peripheral immune system in PD is in part driven by pathogenic forms of alpha synuclein in peripheral tissue. ${ }^{86}$ This can exacerbate inflammation and neurodegenerative pathology within the brain and contribute to more rapid clinical progression. ${ }^{87}$ Azathioprine, an immunosuppressant drug, is widely used in clinical practice for a range of immune-related 
conditions. ${ }^{88}$ It's well established efficacy as an immunosuppressive drug, acceptable safety profile, ease of administration and availability of well-established protocols for monitoring have led to it being explored in PD. A 'proof of concept' study (https://ccpp.cam.ac.uk/ClinicalTrials/clinical-trial-b-azathioprine) aiming to open for recruitment in 2021 will explore whether immunosuppression in early PD with azathioprine has an effect on the subsequent disease course over 12 months and if this is maintained over the subsequent 6 months after drug withdrawal. ${ }^{89}$ This trial will also explore the impact of azathioprine on blood, CSF and neuroimaging parameters of immune activation. To maximise the likelihood of detecting clinical effects with only 1 year follow up, patients predicted to have rapidly progressive disease will be recruited..$^{90}$

\section{Mitochondria}

Mitochondrial dysfunction is seen in sporadic PD, mitochondrial toxins can cause acute emergence of parkinsonism, and it is becoming clear that accumulation of alpha synuclein in mitochondria impairs mitochondrial function and can lead to cell death. ${ }^{91}$

Encouraging observations of "mitochondrial approaches" in dopaminergic-toxin models of PD have thus far been followed by disappointments in the clinic, reinforcing the need for better levels of preclinical evidence. A recent example of this is pioglitazone. Despite showing promise in animal models in reducing neurodegeneration via improved mitochondrial biogenesis, ${ }^{92}$ pioglitazone was not effective in slowing PD progression in a phase II, multicentre, double- blinded RCT. ${ }^{93}$ Similarly, while urate (an anti-oxidant) seemed to prevent dopaminergic neuronal loss in a rat model of PD, and inosine was shown to be effective in raising serum and CSF levels of urate ${ }^{94}$ the 24 month SURE- PD3, phase III RCT failed to find any evidence of neuroprotection from inosine, despite targeting recruitment towards the 
subgroup of patients with low urate levels at baseline. The failure of these studies could in part be related to the poor predictability of effectiveness of the inflammatory ${ }^{92}$ or the dopaminergic toxin based animal models ${ }^{95}$ to the broader population of PD patients subsequently included in trials and a pure reliance on clinical outcome measures to demonstrate disease modification.

Coenzyme Q10 should also facilitate mitochondrial bioenergetics and yet failed to demonstrate efficacy in a large trial of individuals with PD. ${ }^{96}$ There is ongoing interest in testing coenzyme Q10 among a more refined cohort of individuals e.g. parkin mutations, where the underlying pathogenetic process is more clearly linked to mitochondrial dysfunction but data on target engagement will be essential ${ }^{97}$.

Mitochondrial dysfunction has been implicated in PD pathogenesis not only in people with mitochondrial mutations (such as parkin) but also in patients with LRRK2 mutations. Using cells from skin biopsies taken from these patients, and following a screen of 2000 compounds licensed for use in humans, ursodeoxycholic acid (UDCA), a drug used for the management of primary biliary cholangitis, emerged as the most promising agent with the potential to improve mitochondrial function. ${ }^{98,15}$ The mitochondrial rescue potential of UDCA has now also been demonstrated in peripheral tissue of patients with sporadic $\mathrm{PD}^{14}$. This drug has good CNS penetration and is potentially neuroprotective in other neurological conditions. ${ }^{99}$ Two trials to investigate its safety and efficacy data have commenced in $\mathrm{PD}^{100}$ (NCT03840005). Both trials will also measure ATP levels using ${ }^{31} \mathrm{P}$ MR spectroscopy to confirm target engagement, one of them using 7T to quantify energy metabolites in the occipital lobe, the other study $3 \mathrm{~T}$ to determine target engagement in the substantia nigra itself.

Insulin \& GLP1 
Glucagon-like peptide-1 (GLP-1) receptor agonists are widely used for the treatment of type 2 diabetes. These agents stimulate glucose level-dependent insulin release, and $\beta$ islet cell proliferation. GLP-1 receptors exist in the brain and agonists were found to be beneficial across the whole range of dopaminergic and alpha-synuclein animal models via a variety of potential mechanisms including reducing inflammation ${ }^{101}$ and the accumulation of alpha-synuclein. ${ }^{102}$ Exenatide is the most widely tested of these agents and was found to provide both motor and cognitive improvements in an open label Phase II study ${ }^{103}$ with beneficial effects persisting 12 months after drug cessation. ${ }^{104}$ A subsequent randomized, double-blind Phase II trial found a similar improvement in motor outcomes after 48 weeks exposure that was prolonged albeit at a lower level 12 weeks after drug withdrawal raising the question regarding whether it has novel symptomatic or disease modifying effects. ${ }^{105}$ To further explore target engagement, blood samples were collected longitudinally throughout the trial, from which neuronal derived extracellular vesicles were separated. Analysis of these vesicles suggested changes in the insulin signalling, Akt and mTOR pathway that closely mirrored the clinical scores. ${ }^{106}$

A Phase III clinical trial of exenatide has recently began recruiting (ISRCTN14552789) with results expected in 2024. The trial will follow participants for 2 years to try and distinguish whether previously noted effects are more likely symptomatic or disease modifying, and is also using smartphone assessment of motor function as an exploratory outcome measure. A further open label Phase I clinical trial of exenatide is also underway (https://clinicaltrials.gov/ct2/show/NCT03456687) further exploring whether exenatide leads to changes in free water MRI.

\section{Conclusions and future directions}


PD is associated with a range of pathophysiological processes, including alpha-synuclein aggregation, neuroinflammation, mitochondrial and lysosomal dysfunction, selective neuronal vulnerability, and inadequate compensatory processes that may variably occur at different stages of disease progression. The complexity of these intertwined pathways and the consequent heterogeneity in clinical phenotypes will for some individuals require a targeted approach. Advances in methods to objectively quantify the extent to which an individual may respond to a specific disease modifying approach through objective measures of pathway deficiencies and target engagement should revolutionize our ability to influence disease progression.

Such techniques extend far beyond simple genotyping, which at present will still only identify a small minority of heterogeneity of PD. The use of techniques to measure pathological species of alpha-synuclein, neuroinflammation, mitochondrial and lysosomal dysfunction and even an individual's gut microbiome will enable better prediction of drug responders and nonresponders. Agents which have actions downstream from all of these processes should have relevance for the majority of patients with $\mathrm{PD}$, and therefore are less dependent on refinements in patient selection, and of greater impact on the population of PD patients as a whole.

Careful consideration of early phase trial designs that are able to quickly and cost effectively detect evidence of target engagement and indicators confirming a strong likelihood of clinical efficacy will enable agents to be rapidly considered as futile or worthy of evaluation in long term clinical trials. Targeted therapeutics with patient selection based on identification of their primary pathway of disease biology will emerge. Multiple arm, adaptive trial designs will likely soon become the normal way of assessing novel agents in PD and will need to consider stratification issues. There is much scope to improve on our existing clinical outcome 
measures, and remote monitoring, artificial intelligence and sensor quantification of motor and non-motor symptoms will greatly enhance the ultimate arbiter of success- i.e. self-reporting of patient quality of life.

\section{Legends to Figures}

Figure 1 summarises the main neuronal targets for disease modifying interventions in PD. Targets can broadly be divided into precision and broad approaches. Precision approaches target specific organelles (mitochondria, lysosomes) and/or associated proteins (Gcase, LRRK2) with unique downstream molecular influences on common pathological proteins such as $\alpha$-synuclein and apply to a specific subgroup of PD patients with aberrancies in these respective aspects. Broad approaches outlined target a variety of neuronal abnormalities that have been shown to be aberrant in the majority of PD patients. These include therapies specifically targeting $\alpha$-synuclein production ( $\mathrm{B}_{2}$-AR, ASO strategies) and cell to cell transmission as well as therapies targeting other molecular pathways implicated in neuronal survival (GLP-1, Calcium, Iron, Neuroinflammation). Abbreviations $\alpha$-syn / $\alpha$-synuclein; alpha-synuclein, GCase; Glucocerebrosidase, GLP-1; Glucagon-like peptide-1, LRRK-2; Leucine-rich repeat kinase 2 .

Figure 2 summarises potential trial designs that could be employed to improve detection of disease modification effects. All trial approaches outlined provide for a second intervention period to better distinguish symptomatic effects from disease modification effects. Figure 2A depicts a washout trial design. Patients are randomized to either receive placebo or the active treatment in period 1 . In period 2 both the active drug and placebo are withdrawn. If a significant disability gap is noted between groups at the end of an adequately long period 2 this supports the drug having a disease modification effect (black line). Figure 2B depicts a delayed start trial design. Patients are randomized to either receive placebo or the active treatment in period 1 . The group receiving the active treatment in period 1 is defined as the early start group. In period 2, the group receiving placebo in period 1 also receives the active treatment (delayed start group). If a significant disability gap is noted between the groups at the end of period 2 this suggests a disease modification effect (black line). Figure 2C depicts a randomized withdrawal trial design. In period 1 patients are randomised to either receive placebo or active treatment. Period 2 consists of 3 arms. The group receiving placebo in period 1 continue to receive placebo in period 2 while patients who received the active treatment in period 1 will be randomised to either receive placebo or the active treatment. This can be applied to all patients who received the active treatment in period 1 (randomization withdrawal of all patients trial design) or only those who responded to the active treatment in period 1 (randomization withdrawal of responders trial design). If a significant disability gap is noted between the group that received placebo in both periods and those who received the active treatment in period 1 but placebo in period 2, this suggests a disease modification effect. Figure 2D depicts a long-term trial design. Long-term study designs allow for a more prolonged period of assessing disability, often years. In a simple long-term study, patients are randomized to either receive the active treatment or placebo and observed for a long period. In long-term studies with more complex protocols, symptomatic treatments are not permitted in period 1 and introduced in period 2 in both the placebo and 
active groups. With an open protocol, a predefined milestone is the trigger for initiation of symptomatic treatment. If the time lapse before which this occurs is significantly greater in the active treatment group this potentially reflects a disease modification effect. With a strict protocol approach, patients in both groups in period 1 are not allowed to take symptomatic medications for a fixed length of time. During period 2, patients continue to stay in their study groups but are given a standardized symptomatic regimen. If a significant disability gap is noted in period 2 between both the placebo and active treatment groups despite the addition of standardized symptomatic treatment, this suggests a disease modification effect.

\section{Author Contributions:}

NV screened the results of the literature searches for relevant articles. All authors equally contributed to the writing of the manuscript.

\section{Acknowledgement:}

NV's research time and position is funded by the Janet Owens charitable foundation.

\section{Declaration of interests:}

NV has received educational support from Abbvie, Stada, Ipsen and Merc, speaker's honorarium from Abbvie \& Stada and served on advisory boards for Abbvie \& Britannia Pharmaceuticals.

Tanya Simuni has served as a consultant for Acadia, Abbvie, Accorda, Adamas, Allergan, Amneal, Aptinyx, Denali, General Electric (GE), Kyowa, Neuroderm, Neurocrine, Sanofi, Sinopia, Sunovion, Roche, Takeda, Voyager, US World Meds, Parkinson's Foundation, and the Michael J. Fox Foundation for Parkinson's Research; Dr. Simuni has served as a speaker and received an honorarium from Acadia and Adamas; Dr Simuni is on the Scientific advisory board for Neuroderm and Sanofi. Dr. Simuni has received research funding from the NINDS, 
Parkinson's Foundation, MJFF, Biogen, Roche, Neuroderm, Sanofi, Sun Pharma, Abbvie, IMPAX and Prevail.

Professor Huw Morris is employed by UCL. In the last 24 months he reports paid consultancy from Biogen, UCB, Abbvie, Denali, Biohaven, Lundbeck; lecture fees/honoraria from Biogen, UCB, C4X Discovery, GE-Healthcare, Wellcome Trust, Movement Disorders Society; Research Grants from Parkinson's UK, Cure Parkinson's Trust, PSP Association, CBD Solutions, Drake Foundation, Medical Research Council. Professor Morris is a coapplicant on a patent application related to C9ORF72 - Method for diagnosing a neurodegenerative disease (PCT/GB2012/052140).

OB has received grant support from the JP Moulton Charitable Trust, Cure Parkinson's Trust, Michael J Fox Foundation, the Medical Research Council and additional financial support from NZP UK Ltd.

TF has received grant support form Cure Parkinson's trust, Michael J Fox Foundation, John Black Charitable Foundation, Van Andel Institute, Defeat MSA, Innovate UK, National Institute for Health Research \& the Edmond J Safra Foundation. He has received honoraria for speaking at meetings sponsored by Bial, Profile Pharma, Britannia and Boston Scientific. He has served on advisory boards for Living Cell Technologies, Handl, Voyager Therapeutics and Oxford Biomedica.

\section{References:}


1 Ray Dorsey E, Elbaz A, Nichols E, et al. Global, regional, and national burden of Parkinson's disease, 1990-2016: a systematic analysis for the Global Burden of Disease Study 2016. Lancet Neurol 2018; 17: 939-53.

2 Kalia L V., Lang AE. Parkinson’s disease. Lancet. 2015; 386: 896-912.

3 McFarthing K, Buff S, Rafaloff G, Dominey T, Wyse RK, Stott SRW. Parkinson's Disease Drug Therapies in the Clinical Trial Pipeline: 2020. J Parkinsons Dis 2020; 10: $757-74$.

4 Barker RA, Björklund A, Gash DM, et al. GDNF and Parkinson's Disease: Where Next? A Summary from a Recent Workshop. In: Journal of Parkinson's Disease. 2020: 10(3):875-891.

5 Espay AJ, Kalia L V., Gan-Or Z, et al. Disease modification and biomarker development in Parkinson disease: Revision or reconstruction? Neurology 2020; 94: 481-94.

6 Nguyen M, Wong YC, Ysselstein D, Severino A, Krainc D. Synaptic, Mitochondrial, and Lysosomal Dysfunction in Parkinson's Disease. Trends Neurosci 2019; 42: 140-9.

7 Brown EG, Goldman SM. Modulation of the Microbiome in Parkinson's Disease: Diet, Drug, Stool Transplant, and Beyond. Neurotherapeutics 2020; 17: 1406-17.

8 Bezard E, Yue Z, Kirik D, Spillantini MG. Animal models of Parkinson's disease: Limits and relevance to neuroprotection studies. Mov. Disord. 2013; 28: 61-70.

9 Duty S, Jenner P. Animal models of Parkinson's disease: A source of novel treatments and clues to the cause of the disease. Br. J. Pharmacol. 2011; 164: 1357-91.

10 Ekstrand MI, Galter D. The MitoPark Mouse - An animal model of Parkinson's disease with impaired respiratory chain function in dopamine neurons. Park Relat Disord 2009; 15: S185-8.

11 Volpicelli-Daley LA, Luk KC, Patel TP, et al. Exogenous $\alpha$-Synuclein Fibrils Induce 
Lewy Body Pathology Leading to Synaptic Dysfunction and Neuron Death. Neuron 2011; 72: 57-71.

12 Luk KC, Lee VMY. Modeling Lewy pathology propagation in Parkinson's disease. Park Relat Disord 2014; 20: S85-7.

13 Burbulla LF, Song P, Mazzulli JR, et al. Dopamine oxidation mediates mitochondrial and lysosomal dysfunction in Parkinson's disease. Science (80- ) 2017; 357: 1255-61.

14 Carling PJ, Mortiboys H, Green C, et al. Deep phenotyping of peripheral tissue facilitates mechanistic disease stratification in sporadic Parkinson's disease. Prog Neurobiol 2020; 187: 101772.

15 Mortiboys H, Aasly J, Bandmann O. Ursocholanic acid rescues mitochondrial function in common forms of familial Parkinson's disease. Brain 2013; 136: 3038-50.

16 Smits LM, Schwamborn JC. Midbrain Organoids: A New Tool to Investigate Parkinson's Disease. Front. Cell Dev. Biol. 2020; 8: 359.

17 Naoi M, Maruyama W, Shamoto-Nagai M. Rasagiline and selegiline modulate mitochondrial homeostasis, intervene apoptosis system and mitigate $\alpha$-synuclein cytotoxicity in disease-modifying therapy for Parkinson's disease. J. Neural Transm. 2020; 127: 131-47.

18 Rascol O, Fitzer-Attas CJ, Hauser R, et al. A double-blind, delayed-start trial of rasagiline in Parkinson's disease (the ADAGIO study): prespecified and post-hoc analyses of the need for additional therapies, changes in UPDRS scores, and nonmotor outcomes. Lancet Neurol 2011; 10: 415-23.

19 Mitchell T, Archer DB, Chu WT, et al. Neurite orientation dispersion and density imaging (NODDI) and free-water imaging in Parkinsonism. Hum Brain Mapp 2019; 40: $5094-107$.

20 Mollenhauer B, Caspell-Garcia CJ, Coffey CS, et al. Longitudinal analyses of 
cerebrospinal fluid $\alpha$-Synuclein in prodromal and early Parkinson's disease. Mov Disord 2019; 34: 1354-64.

21 Eberling JL, Dave KD, Frasier MA. $\alpha$-synuclein Imaging: A Critical Need for Parkinson's Disease Research. J Parkinsons Dis 2013; 3: 565-7.

22 Alcalay RN, Levy OA, Waters CC, et al. Glucocerebrosidase activity in Parkinson's disease with and without GBA mutations. Brain 2015; 138: 2648-58.

23 Rango M, Dossi G, Squarcina L, Bonifati C. Brain Mitochondrial Impairment in Early-Onset Parkinson's Disease With or Without PINK1 Mutation. Mov Disord 2020; 35: 504-7.

24 Arora S, Baig F, Lo C, et al. Smartphone motor testing to distinguish idiopathic REM sleep behavior disorder, controls, and PD. Neurology 2018; 91: e1528-38.

25 Espay AJ, Bonato P, Nahab FB, et al. Technology in Parkinson's disease: Challenges and opportunities. Mov Disord 2016; 31: 1272-82.

26 Fahn S, Oakes D, Shoulson I, et al. Levodopa and the progression of parkinson's disease. N Engl J Med 2004; 351: 2498-508.

27 Verschuur CVM, Suwijn SR, Boel JA, et al. Randomized delayed-start trial of levodopa in Parkinson's disease. N Engl J Med 2019; 380: 315-24.

28 Thibault L, Rascol O, Corvol JC, et al. New perspectives on study designs for evaluating neuroprotection in Parkinson's disease. Mov Disord 2017; 32: 1365-70.

29 Elm JJ, Hauser R, Tilley BC, et al. Design innovations and baseline findings in a longterm parkinson's trial: The national institute of neurological disorders and stroke exploratory trials in parkinson's disease long-term study-1. Mov Disord 2012; 27: $1513-21$.

30 Kieburtz K, Katz R, McGarry A, Olanow CW. A New Approach to the Development of Disease-Modifying Therapies for PD; Fighting Another Pandemic. Mov Disord 
2021; 36: 59-63.

31 Engelender S, Isacson O. The Threshold Theory for Parkinson's Disease. Trends Neurosci. 2017; 40: 4-14.

32 Chartier-Harlin MC, Kachergus J, Roumier C, et al. $\alpha$-synuclein locus duplication as a cause of familial Parkinson's disease. Lancet 2004; 364: 1167-9.

33 Paumier KL, Luk KC, Manfredsson FP, et al. Intrastriatal injection of pre-formed mouse $\alpha$-synuclein fibrils into rats triggers $\alpha$-synuclein pathology and bilateral nigrostriatal degeneration. Neurobiol Dis 2015; 82: 185-99.

34 Mao X, Ou MT, Karuppagounder SS, et al. Pathological $\alpha$-synuclein transmission initiated by binding lymphocyte-activation gene 3. Science (80- ) 2016; 353: aah3374.

35 Kordower JH, Chu Y, Hauser RA, Freeman TB, Olanow CW. Lewy body-like pathology in long-term embryonic nigral transplants in Parkinson's disease. Nat Med 2008; 14: 504-6.

36 Braak H, Rüb U, Gai WP, Del Tredici K. Idiopathic Parkinson's disease: Possible routes by which vulnerable neuronal types may be subject to neuroinvasion by an unknown pathogen. J Neural Transm 2003; 110: 517-36.

37 Chen Z, Yang Y, Yang X, et al. Immune effects of optimized DNA vaccine and protective effects in a MPTP model of Parkinson's disease. Neurol Sci 2013; 34: $1559-70$.

38 Sanchez-Guajardo V, Annibali A, Jensen PH, Romero-Ramos M. $\alpha$-synuclein vaccination prevents the accumulation of parkinson disease-like pathologic inclusions in striatum in association with regulatory $\mathrm{T}$ cell recruitment in a rat model. $J$ Neuropathol Exp Neurol 2013; 72: 624-45.

39 Schneeberger A, Tierney L, Mandler M. Active immunization therapies for Parkinson's disease and multiple system atrophy. Mov. Disord. 2016; 31: 214-24. 
40 Bergström AL, Kallunki P, Fog K. Development of Passive Immunotherapies for Synucleinopathies. Mov. Disord. 2016; 31: 203-13.

41 Schenk DB, Koller M, Ness DK, et al. First-in-human assessment of PRX002, an anti$\alpha$-synuclein monoclonal antibody, in healthy volunteers. Mov Disord 2017; 32: 211-8.

42 Mittal S, Bjørnevik K, Im DS, et al. $\beta 2$-Adrenoreceptor is a regulator of the $\alpha$ synuclein gene driving risk of Parkinson's disease. Science (80- ) 2017; 357: 891-8.

43 Searles Nielsen S, Gross A, Camacho-Soto A, Willis AW, Racette BA. $\beta 2$ adrenoreceptor medications and risk of Parkinson disease. Ann Neurol 2018; 84: 68393.

44 Alexander GM, Schwartzman RJ, Nukes TA, Grothusen JR, Hooker MD. $\beta 2$ Adrenergic agonist as adjunct therapy to levodopa in Parkinson's disease. Neurology 1994; 44: 1511-3.

45 Uc EY, Lambert CP, Harik SI, Rodnitzky RL, Evans WJ. Albuterol improves response to levodopa and increases skeletal muscle mass in patients with fluctuating Parkinson disease. Clin Neuropharmacol 2003; 26: 207-12.

46 Fowler AJ, Moussa CEH. Activating Autophagy as a Therapeutic Strategy for Parkinson's Disease. CNS Drugs 2018; 32: 1-11.

47 Hantschel O, Superti-Furga G. Regulation of the c-Abl and Bcr-Abl tyrosine kinases. Nat. Rev. Mol. Cell Biol. 2004; 5: 33-44.

48 Hebron ML, Lonskaya I, Moussa CEH. Nilotinib reverses loss of dopamine neurons and improvesmotorbehavior via autophagic degradation of $\alpha$-synuclein in parkinson's disease models. Hum Mol Genet 2013; 22: 3315-28.

49 Pagan F, Hebron M, Valadez EH, et al. Nilotinib effects in Parkinson's disease and dementia with lewy bodies. J Parkinsons Dis 2016; 6: 503-17.

50 Pagan FL, Hebron ML, Wilmarth B, et al. Pharmacokinetics and pharmacodynamics 
of a single dose Nilotinib in individuals with Parkinson's disease. Pharmacol Res Perspect 2019; 7: e00470.

51 Simuni T, Kostrzebski M, Fiske B, et al. Abstract book of the 5th World Parkinson Congress, Kyoto, Japan, June 4-7, 2019. J Parkinsons Dis 2019; 9: 1-278.

52 Orenstein SJ, Kuo S-H, Tasset I, et al. Interplay of LRRK2 with chaperone-mediated autophagy. Nat Neurosci 2013; 16: 394-406.

53 Nalls MA, Blauwendraat C, Vallerga CL, et al. Identification of novel risk loci, causal insights, and heritable risk for Parkinson's disease: a meta-analysis of genome-wide association studies. Lancet Neurol 2019; 18: 1091-102.

54 Cresto N, Gardier C, Gubinelli F, et al. The unlikely partnership between LRRK2 and $\alpha$-synuclein in Parkinson's disease. Eur. J. Neurosci. 2019; 49: 339-63.

55 Lee BD, Shin JH, Vankampen J, et al. Inhibitors of leucine-rich repeat kinase-2 protect against models of Parkinson's disease. Nat Med 2010; 16: 998-1000.

56 Manzoni C, Mamais A, Dihanich S, et al. Inhibition of LRRK2 kinase activity stimulates macroautophagy. Biochim Biophys Acta - Mol Cell Res 2013; 1833: 290010.

57 Di Maio R, Hoffman EK, Rocha EM, et al. LRRK2 activation in idiopathic Parkinson's disease. Sci Transl Med 2018; 10: eaar5429.

58 Zhao HT, John N, Delic V, et al. LRRK2 Antisense Oligonucleotides Ameliorate $\alpha$ Synuclein Inclusion Formation in a Parkinson's Disease Mouse Model. Mol Ther Nucleic Acids 2017; 8: 508-19.

59 Korecka JA, Thomas R, Hinrich AJ, et al. Splice-Switching Antisense Oligonucleotides Reduce LRRK2 Kinase Activity in Human LRRK2 Transgenic Mice. Mol Ther - Nucleic Acids 2020; 4: 623-35.

60 Sidransky E, Nalls MA, Aasly JO, et al. Multicenter analysis of glucocerebrosidase 
mutations in Parkinson's disease. N Engl J Med 2009; 361: 1651-61.

61 Do J, McKinney C, Sharma P, Sidransky E. Glucocerebrosidase and its relevance to Parkinson disease. Mol. Neurodegener. 2019; 14: 36.

62 McNeill A, Magalhaes J, Shen C, et al. Ambroxol improves lysosomal biochemistry in glucocerebrosidase mutation-linked Parkinson disease cells. Brain 2014; 137: 148195.

63 Mullin S, Smith L, Lee K, et al. Ambroxol for the Treatment of Patients with Parkinson Disease with and Without Glucocerebrosidase Gene Mutations: A Nonrandomized, Noncontrolled Trial. JAMA Neurol 2020; 77: 427-34.

64 Aflaki E, Borger DK, Moaven N, et al. A new glucocerebrosidase chaperone reduces $\alpha$-synuclein and glycolipid levels in iPSC-derived dopaminergic neurons from patients with Gaucher disease and parkinsonism. J Neurosci 2016; 36: 7441-52.

65 Rocha EM, Smith GA, Park E, et al. Glucocerebrosidase gene therapy prevents $\alpha$ synucleinopathy of midbrain dopamine neurons. Neurobiol Dis 2015; 82: 495-503.

66 Sardi SP, Viel C, Clarke J, et al. Glucosylceramide synthase inhibition alleviates aberrations in synucleinopathy models. Proc Natl Acad Sci U S A 2017; 114: 2699 704.

67 Gan-Or Z, Liong C, Alcalay RN. GBA-Associated Parkinson's Disease and Other Synucleinopathies. Curr. Neurol. Neurosci. Rep. 2017; 18: 44.

68 Chan CS, Guzman JN, Ilijic E, et al. 'Rejuvenation' protects neurons in mouse models of Parkinson's disease. Nature 2007; 447: 1081-6.

69 Ilijic E, Guzman JN, Surmeier DJ. The L-type channel antagonist isradipine is neuroprotective in a mouse model of Parkinson's disease. Neurobiol Dis 2011; 43: $364-71$.

70 Becker C, Jick SS, Meier CR. Use of antihypertensives and the risk of Parkinson 
disease. Neurology 2008; 70: 1438-44.

71 Isradipine Versus Placebo in Early Parkinson Disease: A Randomized Trial. Ann Intern Med 2020; 172: 591-8.

72 Dexter DT, Wells FR, Agid F, et al. INCREASED NIGRAL IRON CONTENT IN POSTMORTEM PARKINSONIAN BRAIN. Lancet. 1987; 330: 1219-20.

73 Langkammer C, Pirpamer L, Seiler S, et al. Quantitative susceptibility mapping in Parkinson's disease. PLoS One 2016; 11: e0162460.

74 Martin-Bastida A, Ward RJ, Newbould R, et al. Brain iron chelation by deferiprone in a phase 2 randomised double-blinded placebo controlled clinical trial in Parkinson's disease. Sci Rep 2017; 7: 1398.

75 International Parkinson's Disease Genomics Consortium I, Wellcome Trust Case Control W. A Two-Stage Meta-Analysis identifies several new loci for Parkinson's Disease. PLoS Genet 2011; 7: e1002142.

76 Halliday GM, Stevens CH. Glia: Initiators and progressors of pathology in Parkinson's disease. Mov. Disord. 2011; 26: 6-17.

77 Imamura K, Hishikawa N, Sawada M, Nagatsu T, Yoshida M, Hashizume Y. Distribution of major histocompatibility complex class II-positive microglia and cytokine profile of Parkinson's disease brains. Acta Neuropathol 2003; 106: 518-26.

78 Gendelman HE, Zhang Y, Santamaria P, et al. Evaluation of the safety and immunomodulatory effects of sargramostim in a randomized, double-blind phase 1 clinical Parkinson's disease trial. npj Park Dis 2017; 3: 10.

79 Gellhaar S, Sunnemark D, Eriksson H, Olson L, Galter D. Myeloperoxidaseimmunoreactive cells are significantly increased in brain areas affected by neurodegeneration in Parkinson's and Alzheimer's disease. Cell Tissue Res 2017; 369: $445-54$. 
80 Posener JA, Hauser RA, Stieber M, et al. Safety, tolerability, and pharmacodynamics of AZD3241, a myeloperoxidase inhibitor, in Parkinson's disease. Mov Disord 2014; 29: S259-60.

81 Jucaite A, Svenningsson P, Rinne JO, et al. Effect of the myeloperoxidase inhibitor AZD3241 on microglia: A PET study in Parkinson's disease. Brain 2015; 138: $2687-$ 700.

82 Codolo G, Plotegher N, Pozzobon T, et al. Triggering of Inflammasome by Aggregated $\alpha$-Synuclein, an Inflammatory Response in Synucleinopathies. PLoS One 2013; 8: e55375.

83 Qiao C, Zhang Q, Jiang Q, et al. Inhibition of the hepatic Nlrp3 protects dopaminergic neurons via attenuating systemic inflammation in a MPTP/p mouse model of Parkinson's disease. J Neuroinflammation 2018; 15: 193.

84 Gordon R, Albornoz EA, Christie DC, et al. Inflammasome inhibition prevents synuclein pathology and dopaminergic neurodegeneration in mice. Sci Transl Med 2018; 10: eaah4066.

85 Carroll CB, Wyse RKH. Simvastatin as a Potential Disease-Modifying Therapy for Patients with Parkinson's Disease: Rationale for Clinical Trial, and Current Progress. J. Parkinsons. Dis. 2017; 7: 545-68.

86 Gao HM, Zhang F, Zhou H, Kam W, Wilson B, Hong JS. Neuroinflammation and $\alpha-$ synuclein dysfunction potentiate each other, driving chronic progression of neurodegeneration in a mouse model of Parkinson's disease. Environ Health Perspect 2011; 119: 807-14.

87 Williams-Gray CH, Wijeyekoon R, Yarnall AJ, et al. Serum immune markers and disease progression in an incident Parkinson's disease cohort (ICICLE-PD). Mov Disord 2016; 31: 995-1003. 
88 Broen JCA, van Laar JM. Mycophenolate mofetil, azathioprine and tacrolimus: mechanisms in rheumatology. Nat. Rev. Rheumatol. 2020; 16: 167-78.

89 Greenland JC, Cutting E, Kadyan S, Bond S, Chhabra A, Williams-Gray CH. Azathioprine immunosuppression and disease modification in Parkinson's disease (AZA-PD): a randomised double-blind placebo-controlled phase II trial protocol. $B M J$ Open 2020; 10: e040527.

90 Velseboer DC, De Bie RMA, Wieske L, et al. Development and external validation of a prognostic model in newly diagnosed Parkinson disease. Neurology 2016; 86: 98693.

91 Exner N, Lutz AK, Haass C, Winklhofer KF. Mitochondrial dysfunction in Parkinson's disease: Molecular mechanisms and pathophysiological consequences. EMBO J. 2012; 31: $3038-62$.

92 Pinto M, Nissanka N, Peralta S, Brambilla R, Diaz F, Moraes CT. Pioglitazone ameliorates the phenotype of a novel Parkinson's disease mouse model by reducing neuroinflammation. Mol Neurodegener 2016; 11: 25.

93 Simuni T, Kieburtz K, Tilley B, et al. Pioglitazone in early Parkinson's disease: A phase 2, multicentre, double-blind, randomised trial. Lancet Neurol 2015; 14: 795803.

94 Schwarzschild MA, Ascherio A, Beal MF, et al. Inosine to increase serum and cerebrospinal fluid urate in parkinson disease a randomized clinical trial. JAMA Neurol 2014; 71: 141-50.

95 Gong L, Zhang QL, Zhang N, et al. Neuroprotection by urate on 6-OHDA-lesioned rat model of Parkinson's disease: Linking to Akt/GSK3 $\beta$ signaling pathway. J Neurochem 2012; 123: 876-85.

96 Flint Beal M, Oakes D, Shoulson I, et al. A randomized clinical trial of high-dosage 
coenzyme Q10 in early parkinson disease no evidence of benefit. JAMA Neurol 2014; 75: $543-52$.

97 Prasuhn J, Brüggemann N, Hessler N, et al. An omics-based strategy using coenzyme Q10 in patients with Parkinson's disease: concept evaluation in a double-blind randomized placebo-controlled parallel group trial. Neurol Res Pract 2019; 1.

98 Mortiboys H, Furmston R, Bronstad G, Aasly J, Elliott C, Bandmann O. UDCA exerts beneficial effect on mitochondrial dysfunction in LRRK2 G2019S carriers and in vivo. Neurology 2015; 85: 846-52.

99 Keene CD, Rodrigues CMP, Eich T, Chhabra MS, Steer CJ, Low WC.

Tauroursodeoxycholic acid, a bile acid, is neuroprotective in a transgenic animal model of Huntington's disease. Proc Natl Acad Sci U S A 2002; 99: 10671-6.

100 Sathe AG, Tuite P, Chen C, et al. Pharmacokinetics, Safety, and Tolerability of Orally Administered Ursodeoxycholic Acid in Patients With Parkinson's Disease-A Pilot Study. J Clin Pharmacol 2020; 60: 744-50.

101 Chen S, Yu SJ, Li Y, et al. Post-Treatment with PT302, a long-Acting Exendin-4 sustained release formulation, reduces dopaminergic neurodegeneration in a 6Hydroxydopamine rat model of Parkinson's disease. Sci Rep 2018; 8: 10722.

102 Zhang L, Zhang L, Li L, Hölscher C. Semaglutide is neuroprotective and reduces $\alpha$ synuclein levels in the chronic MPTP mouse model of Parkinson's disease. $J$ Parkinsons Dis 2019; 9: 157-71.

103 Aviles-Olmos I, Dickson J, Kefalopoulou Z, et al. Exenatide and the treatment of patients with Parkinson's disease. J Clin Invest 2013; 123: 2730-6.

104 Aviles-Olmos I, Dickson J, Kefalopoulou Z, et al. Motor and Cognitive Advantages Persist 12 Months after Exenatide Exposure in Parkinson's Disease. J Parkinsons Dis $2015 ; 4: 337-44$. 
105 Athauda D, Maclagan K, Skene SS, et al. Exenatide once weekly versus placebo in Parkinson's disease: a randomised, double-blind, placebo-controlled trial. Lancet 2017; 390: 1664-75.

106 Athauda D, Gulyani S, Karnati HK, et al. Utility of Neuronal-Derived Exosomes to Examine Molecular Mechanisms That Affect Motor Function in Patients with Parkinson Disease: A Secondary Analysis of the Exenatide-PD Trial. JAMA Neurol 2019; 76: 420-9. 